\title{
Global Attractivity and Periodic Solution of a Discrete Multispecies Cooperation and Competition Predator-Prey System
}

\author{
Zheyan Zhou \\ School of Mathematics and Computer Science, Fujian Normal University, Fuzhou 350007, China \\ Correspondence should be addressed to Zheyan Zhou, zheyanzhou@126.com \\ Received 20 April 2011; Accepted 8 June 2011 \\ Academic Editor: Ugurhan Mugan \\ Copyright ( 2011 Zheyan Zhou. This is an open access article distributed under the Creative \\ Commons Attribution License, which permits unrestricted use, distribution, and reproduction in \\ any medium, provided the original work is properly cited. \\ We propose a discrete multispecies cooperation and competition predator-prey systems. For \\ general nonautonomous case, sufficient conditions which ensure the permanence and the global \\ stability of the system are obtained; for periodic case, sufficient conditions which ensure the \\ existence of a globally stable positive periodic solution of the system are obtained.
}

\section{Introduction}

In this paper, we consider the dynamic behavior of the following non-autonomous discrete $n+m$-species cooperation and competition predator-prey systems

$$
\begin{gathered}
x_{i}(k+1)=x_{i}(k) \exp \left[r_{1 i}(k)\left(1-\frac{x_{i}(k)}{a_{i}(k)+\sum_{l=1, l \neq i}^{n} b_{i l}(k) x_{l}(k)}-c_{i}(k) x_{i}(k)\right)\right. \\
\left.-\sum_{l=1}^{m} d_{i l}(k) y_{l}(k)\right], \\
y_{j}(k+1)=y_{j}(k) \exp \left[r_{2 j}(k)+\sum_{l=1}^{n} e_{j l}(k) x_{l}(k)-\sum_{l=1}^{m} p_{j l}(k) y_{l}(k)\right],
\end{gathered}
$$

where $i=1,2, \ldots n ; j=1,2, \ldots, m ; x_{i}(k)$ is the density of prey species $i$ at $k$ th generation. $y_{j}(k)$ is the density of predator species $j$ at $k$ th generation. 
Dynamic behaviors of population models governed by difference equations had been studied by a number of papers, see [1-4] and the references cited therein. It has been found that the autonomous discrete systems can demonstrate quite rich and complicated dynamics, see $[5,6]$. Recently, more and more scholars paid attention to the non-autonomous discrete population models, since such kind of model could be more appropriate.

May [7] suggested the following set of equations to describe a pair of mutualists:

$$
\begin{aligned}
& \dot{u}=r_{1} u\left(1-\frac{u}{a_{1}+b_{1} v}-c_{1} u\right), \\
& \dot{v}=r_{2} v\left(1-\frac{v}{a_{2}+b_{2} u}-c_{2} v\right),
\end{aligned}
$$

where $u, v$ are the densities of the species $U, V$ at time $t$, respectively. $r_{i}, a_{i}, b_{i}, c_{i}, i=1,2$ are positive constants. He showed that system (1.2) has a globally asymptotically stable equilibrium point in the region $u>0, v>0$.

Bai et al. [8] argued that the discrete case of cooperative system is more appropriate, and they proposed the following system:

$$
\begin{aligned}
& x_{1}(k+1)=x_{1}(k) \exp \left\{r_{1}(k)\left[1-\frac{x_{1}(k)}{a_{1}(k)+b_{1}(k) x_{2}(k)}-c_{1}(k) x_{1}(k)\right]\right\} \\
& x_{2}(k+1)=x_{2}(k) \exp \left\{r_{2}(k)\left[1-\frac{x_{2}(k)}{a_{2}(k)+b_{2}(k) x_{2}(k)}-c_{2}(k) x_{1}(k)\right]\right\} .
\end{aligned}
$$

Chen [9] investigated the dynamic behavior of the following discrete $n+m$-species Lotka-Volterra competition predator-prey systems

$$
\begin{aligned}
& x_{i}(k+1)=x_{i}(k) \exp \left[b_{i}(k)-\sum_{l=1}^{n} a_{i l}(k) x_{l}(k)-\sum_{l=1}^{m} c_{i l}(k) y_{l}(k)\right], \\
& y_{j}(k+1)=y_{j}(k) \exp \left[r_{j}(k)+\sum_{l=1}^{n} d_{j l}(k) x_{l}(k)-\sum_{l=1}^{m} e_{j l}(k) y_{l}(k)\right],
\end{aligned}
$$

he investigated the dynamic behavior of the system (1.4).

The aim of this paper is, by further developing the analysis technique of Huo and $\mathrm{Li}$ [10] and Chen [9], to obtain a set of sufficient condition which ensure the permanence and the global stability of the system (1.1); for periodic case, sufficient conditions which ensure the existence of a globally stable positive periodic solution of the system (1.1) are obtained.

We say that system (1.1) is permanent if there are positive constants $M$ and $m$ such that for each positive solution $\left(x_{1}(k), \ldots, x_{n}(k), y_{1}(k), \ldots, y_{m}(k)\right)$ of system (1.1) satisfies

$$
\begin{aligned}
& m \leq \lim _{k \rightarrow+\infty} \inf x_{i}(k) \leq \lim _{k \rightarrow+\infty} \sup x_{i}(k) \leq M \\
& m \leq \lim _{k \rightarrow+\infty} \inf y_{i}(k) \leq \lim _{k \rightarrow+\infty} \sup y_{i}(k) \leq M
\end{aligned}
$$

for all $i=1,2, \ldots, n ; j=1,2, \ldots, m$. 
Throughout this paper, we assume that $r_{1 i}(k), b_{i l}(k), a_{i}(k), c_{i}(k), r_{2 j}(k), d_{i l}(k), e_{j l}(k)$, $p_{j l}(k)$ are all bounded nonnegative sequence, and use the following notations for any bounded sequence $\{a(k)\}$

$$
a^{u}=\sup _{k \in N} a(k), \quad a^{l}=\inf _{k \in N} a(k) .
$$

with

For biological reasons, we only consider solution $\left(x_{1}(k), \ldots, x_{n}(k), y_{1}(k), \ldots, y_{m}(k)\right)$

$$
x_{i}(0)>0 ; \quad i=1,2, \ldots, n, \quad y_{j}(0)>0, \quad j=1,2, \ldots, m .
$$

Then system (1.1) has a positive solution $\left(x_{1}(k), \ldots, x_{n}(k), y_{1}(k), \ldots, y_{m}(k)\right)_{k=0}^{\infty}$ passing through $\left(x_{1}(0), \ldots, x_{n}(0), y_{1}(0), \ldots, y_{m}(0)\right)$.

The organization of this paper is as follows. In Section 2, we obtain sufficient conditions which guarantee the permanence of the system (1.1). In Section 3, we obtain sufficient conditions which guarantee the global stability of the positive solution of system (1.1). As a consequence, for periodic case, we obtain sufficient conditions which ensure the existence of a globally stable positive solution of system (1.1).

\section{Permanence}

In this section, we establish permanence results for system (1.1).

Lemma 2.1 (see [11]). Let $k \in N_{k_{0}}^{+}=\left\{k_{0}, k_{0}+1, \ldots, k_{0}+l, \ldots\right\}, r \geq 0$. For any fixed $k, g(k, r)$ is a non-decreasing function with respect to $r$, and for $k \geq k_{0}$, the following inequalities hold:

$$
\begin{aligned}
& y(k+1) \leq g(k, y(k)), \\
& u(k+1) \geq g(k, u(k)) .
\end{aligned}
$$

If $y\left(k_{0}\right) \leq u\left(k_{0}\right)$, then $y(k) \leq u(k)$ for all $k \geq k_{0}$.

Now let one consider the following single species discrete model:

$$
N(k+1)=N(k) \exp \{a(k)-b(k) N(k)\},
$$

where $\{a(k)\}$ and $\{b(k)\}$ are strictly positive sequences of real numbers defined for $k \in N=$ $\{0,1,2, \ldots\}$ and $0<a^{l} \leq a^{u}, 0<b^{l} \leq b^{u}$.

Lemma 2.2 (see [12]). Any solution of system (2.5) with initial condition $N(0)>0$ satisfies

$$
m \leq \lim _{k \rightarrow+\infty} \inf N(k) \leq \lim _{k \rightarrow+\infty} \sup N(k) \leq M
$$


where

$$
M=\frac{1}{b^{l}} \exp \left\{a^{u}-1\right\}, \quad m=\frac{a^{l}}{b^{u}} \exp \left\{a^{l}-b^{u} M\right\} .
$$

Proposition 2.3. Assume that

$$
-r_{2 j}^{u}+\sum_{l=1}^{n} e_{j l}^{l} M_{l}>0, \quad j=1,2, \ldots, m
$$

holds, then

$$
\begin{aligned}
& \lim _{k \rightarrow+\infty} \sup x_{i}(k) \leq M_{i}, \quad i=1,2, \ldots, n, \\
& \lim _{k \rightarrow+\infty} \sup y_{i}(k) \leq Q_{i}, \quad i=1,2, \ldots, m,
\end{aligned}
$$

where

$$
\begin{gathered}
M_{i}=\frac{1}{c_{i}^{l} r_{1 i}^{l}} \exp \left\{r_{1 i}^{u}-1\right\}, \\
Q_{i}=\frac{1}{p_{i i}^{l}} \exp \left\{\sum_{l=1}^{n} e_{i l}^{u} M_{l}-r_{2 i}^{l}-1\right\} .
\end{gathered}
$$

Proof. Let $u(k)=\left(x_{1}(k), \ldots, x_{n}(k), y_{1}(k), \ldots, y_{m}(k)\right)$ be any positive solution of system (1.1), from the $i$ th equation of (1.1) we have

$$
\begin{aligned}
x_{i}(k+1) & \leq x_{i}(k) \exp \left\{r_{1 i}(k)\left[1-c_{i}(k) x_{i}(k)\right]\right\} \\
& =x_{i}(k) \exp \left\{r_{1 i}(k)-r_{1 i}(k) c_{i}(k) x_{i}(k)\right\} .
\end{aligned}
$$

By applying Lemmas 2.1 and 2.2, it immediately follows that

$$
\lim _{k \rightarrow+\infty} \sup x_{i}(k) \leq \frac{1}{c_{i}^{l} r_{1 i}^{l}} \exp \left\{r_{1 i}^{u}-1\right\}:=M_{i}
$$

For any positive constant $\varepsilon$ small enough, it follows from (2.9) that there exists enough large $K_{0}$ such that

$$
x_{i}(k) \leq M_{i}+\varepsilon, \quad i=1,2, \ldots, n, \quad \forall k \geq K_{0} .
$$

From the $n+j$ th equation of the system (1.1) and (2.10), we can obtain

$$
y_{j}(k+1) \leq y_{j}(k) \exp \left\{-r_{2 j}(k)+\sum_{l=1}^{n} e_{j l}(k)\left(M_{l}+\varepsilon\right)-p_{j j}(k) y_{j}(k)\right\} .
$$


Condition (2.5) shows that Lemmas 2.1 and 2.2 could be applied to (2.11), and so, by applying Lemmas 2.1 and 2.2, it immediately follows that

$$
\lim _{k \rightarrow+\infty} \sup y_{j}(k) \leq \frac{1}{p_{j j}^{l}} \exp \left\{\sum_{l=1}^{n} e_{j l}^{u}\left(M_{l}+\varepsilon\right)-r_{2 j}^{l}-1\right\}, \quad 1 \leq j \leq m
$$

Setting $\varepsilon \rightarrow 0$ in the above inequality leads to

$$
\lim _{k \rightarrow+\infty} \sup y_{j}(k) \leq \frac{1}{p_{j j}^{l}} \exp \left\{\sum_{l=1}^{n} e_{j l}^{u} M_{l}-r_{2 j}^{l}-1\right\}:=Q_{j}, \quad 1 \leq j \leq m .
$$

This completes the proof of Proposition 2.3.

Now we are in the position of stating the permanence of the system (1.1).

Theorem 2.4. In addition to (2.5), assume further that

$$
\begin{gathered}
r_{1 i}^{l}-\sum_{l=1}^{m} d_{i l}^{u} Q_{l}>0, \quad i=1,2, \ldots, n, \\
-r_{2 j}^{u}+\sum_{l=1}^{n} e_{j l}^{l} m_{l}-\sum_{l=1, l \neq j}^{m} p_{j l}^{u} Q_{l}>0, \quad j=1,2, \ldots m,
\end{gathered}
$$

then system (1.1) is permanent, where

$$
\begin{aligned}
m_{i} & =\frac{r_{1 i}^{l}-\sum_{l=1}^{m} d_{i l}^{u} Q_{l}}{r_{1 i}^{u}\left(1 / a_{i}^{l}+c_{i}^{u}\right)} \exp \left\{r_{1 i}^{l}-\sum_{l=1}^{m} d_{i l}^{u} Q_{l}-r_{1 i}^{u}\left(\frac{1}{a_{i}^{l}}+c_{i}^{u}\right) M_{i}\right\}, \\
Q_{i} & =\frac{1}{p_{i i}^{l}} \exp \left\{\sum_{l=1}^{n} e_{i l}^{u} M_{l}-r_{2 i}^{l}-1\right\} .
\end{aligned}
$$

Proof. By applying Proposition 2.3, we see that to end the proof of Theorem 2.4, it is enough to show that under the conditions of Theorem 2.4,

$$
\begin{aligned}
& \lim _{k \rightarrow+\infty} \inf x_{j}(k) \geq m_{i}, \quad 1 \leq i \leq n, \\
& \lim _{k \rightarrow+\infty} \inf y_{j}(k) \geq q_{j}, \quad 1 \leq j \leq m .
\end{aligned}
$$

From Proposition 2.3, $\forall \varepsilon>0$, there exists a $K_{1}>0, K_{1} \in N, \forall k>K_{1}$,

$$
x_{i}(k) \leq M_{i}+\varepsilon, \quad i=1,2, \ldots, n, \quad y_{j}(k) \leq Q_{j}+\varepsilon, \quad j=1,2, \ldots, m .
$$


From the $i$ th equation of system (1.1) and (2.17), we have

$$
\begin{aligned}
x_{i}(k+1) & \geq x_{i}(k) \exp \left\{r_{1 i}(k)-r_{1 i}(k)\left(\frac{1}{a_{i}^{l}}+c_{i}^{u}\right) x_{i}(k)-\sum_{l=1}^{m} d_{i l}(k)\left(Q_{l}+\varepsilon\right)\right\} \\
& =x_{i}(k) \exp \left\{r_{1 i}(k)-\sum_{l=1}^{m} d_{i l}(k)\left(Q_{l}+\varepsilon\right)-r_{1 i}(k)\left(\frac{1}{a_{i}^{l}}+c_{i}^{u}\right) x_{i}(k)\right\},
\end{aligned}
$$

for all $k>K_{1}$.

By applying Lemmas 2.1 and 2.2 to (2.18), it immediately follows that

$$
\begin{aligned}
\lim _{k \rightarrow+\infty} \inf x_{i}(k) \geq & \frac{r_{1 i}^{l}-\sum_{l=1}^{m} d_{i l}^{u}\left(Q_{l}+\varepsilon\right)}{r_{1 i}^{u}\left(1 / a_{i}^{l}+c_{i}^{u}\right)} \\
& \times \exp \left\{r_{1 i}^{l}-\sum_{l=1}^{m} d_{i l}^{u}\left(Q_{l}+\varepsilon\right)-r_{1 i}^{u}\left(\frac{1}{a_{i}^{l}}+c_{i}^{u}\right) M_{i}\right\} .
\end{aligned}
$$

Setting $\varepsilon \rightarrow 0$ in (2.19) leads to

$$
\begin{aligned}
\lim _{k \rightarrow+\infty} \inf x_{i}(k) \geq & \frac{r_{1 i}^{l}-\sum_{l=1}^{m} d_{i l}^{u} Q_{l}}{r_{1 i}^{u}\left(1 / a_{i}^{l}+c_{i}^{u}\right)} \\
& \times \exp \left\{r_{1 i}^{l}-\sum_{l=1}^{m} d_{i l}^{u} Q_{l}-r_{1 i}^{u}\left(\frac{1}{a_{i}^{l}}+c_{i}^{u}\right) M_{i}\right\}:=m_{i} .
\end{aligned}
$$

Then, for any positive constant $\varepsilon$ small enough, from (2.20) we know that there exists an enough large $K_{2}>K_{1}$ such that

$$
x_{i}(k) \geq m_{i}-\varepsilon, \forall k \geq k_{2} .
$$

Equations (2.17), (2.21) combining with the $n+j$ th equation of the system (1.1) leads to

$$
\begin{aligned}
y_{j}(k+1) \geq y_{j}(k) \exp \left\{-r_{2 j}(k)+\sum_{l=1}^{n} e_{j l}(k)\left(m_{l}-\varepsilon\right)\right. \\
\left.\quad-\sum_{l=1, l \neq j}^{m} p_{j l}(k)\left(Q_{l}+\varepsilon\right)-p_{j j}(k) y_{j}(k)\right\},
\end{aligned}
$$


under the condition (2.14), by applying Lemmas 2.1 and 2.2 to (2.22), it immediately follows that

$$
\begin{aligned}
\lim _{k \rightarrow+\infty} \inf y_{j}(k) \geq & \frac{-r_{2 j}^{u}+\sum_{l=1}^{n} e_{j l}^{l}\left(m_{l}-\varepsilon\right)-\sum_{l=1, l \neq j}^{m} p_{j l}^{u}\left(Q_{l}+\varepsilon\right)}{p_{j j}^{u}} \\
& \times \exp \left\{-r_{2 j}^{u}+\sum_{l=1}^{n} e_{j l}^{l}\left(m_{l}-\varepsilon\right)-\sum_{l=1, l \neq j}^{m} p_{j l}^{u}\left(Q_{l}+\varepsilon\right)-p_{j j}^{u} Q_{j}\right\} .
\end{aligned}
$$

Setting $\varepsilon \rightarrow 0$ in (2.23) leads to

$$
\begin{aligned}
\lim _{k \rightarrow+\infty} \inf y_{j}(k) \geq & \frac{-r_{2 j}^{u}+\sum_{l=1}^{n} e_{j l}^{l} m_{l}-\sum_{l=1, l \neq j}^{m} p_{j l}^{u} Q_{l}}{p_{j j}^{u}} \\
& \times \exp \left\{-r_{2 j}^{u}+\sum_{l=1}^{n} e_{j l}^{l} m_{l}-\sum_{l=1, l \neq j}^{m} p_{j l}^{u} Q_{l}-p_{j j}^{u} Q_{j}\right\}:=q_{j} .
\end{aligned}
$$

This ends the proof of Theorem 2.4.

It should be noticed that, under the assumption of Theorem 2.4, the set

$$
\left[m_{1}, M_{1}\right] \times \cdots\left[m_{n}, M_{n}\right] \times\left[q_{1}, Q_{1}\right] \times \cdots\left[q_{m}, Q_{m}\right]
$$

is an invariant set of system (1.1).

\section{Global Stability}

Now we study the stability property of the positive solution of system (1.1).

Theorem 3.1. Assume that

$$
\begin{aligned}
\lambda_{i}= & \max \left\{\left|1-\left(c_{i}^{u}+\frac{1}{a_{i}^{l}}\right) r_{1 i}^{u} M_{i}\right|,\left|1-\left(c_{i}^{l}+\frac{1}{a_{i}^{u}}\right) r_{1 i}^{l} m_{i}\right|\right\}+\frac{r_{1 i}^{u} M_{i}}{\left(a_{i}^{l}\right)^{2}} \sum_{l=1, l \neq i}^{n} b_{i l}^{u} M_{l} \\
& +\sum_{l=1}^{m} d_{i l}^{u} Q_{l}<1, \\
\delta_{j}= & \max \left\{\left|1-p_{j j}^{u} Q_{j}\right|,\left|1-p_{j j}^{l} q_{j}\right|\right\}+\sum_{l=1}^{n} e_{j l}^{u} M_{l}+\sum_{l=1}^{n} p_{j l}^{u} Q_{l}<1 .
\end{aligned}
$$

Then for any two positive solution $\left(x_{1}(k), \ldots, x_{n}(k), y_{1}(k), \ldots, y_{m}(k)\right)$ and $\left(\tilde{x}_{1}(k), \ldots, \tilde{x}_{n}(k)\right.$, $\left.\tilde{y}_{1}(k), \ldots, \tilde{y}_{m}(k)\right)$ of system (1.1), one has

$$
\lim _{k \rightarrow+\infty}\left(\tilde{x}_{i}(k)-x_{i}(k)\right)=0, \quad \lim _{k \rightarrow+\infty}\left(\tilde{y}_{j}(k)-y_{j}(k)\right)=0 .
$$


Proof. Let

$$
x_{i}(k)=\tilde{x}_{i}(k) \exp \left\{u_{i}(k)\right\}, \quad y_{j}(k)=\tilde{y}_{j}(k) \exp \left\{v_{j}(k)\right\}
$$

Then system (1.1) is equivalent to

$$
\begin{aligned}
u_{i}(k+1)= & u_{i}(k)+\frac{r_{1 i}(k) \tilde{x}_{i}(k)}{a_{i}(k)+\sum_{l=1, l \neq i}^{n} b_{i l}(k) \tilde{x}_{l}(k)}-\frac{r_{1 i}(k) \tilde{x}_{i}(k) \exp \left\{u_{i}(k)\right\}}{a_{i}(k)+\sum_{l=1, l \neq i}^{n} b_{i l}(k) \tilde{x}_{l}(k) \exp \left\{u_{l}(k)\right\}} \\
& -r_{1 i}(k) c_{i}(k) \tilde{x}_{i}(k)\left(\exp \left\{u_{i}(k)\right\}-1\right)-\sum_{l=1}^{m} d_{i l}(k) \tilde{y}_{l}(k)\left(\exp \left\{v_{l}(k)\right\}-1\right), \\
v_{j}(k+1)= & v_{j}(k)-\sum_{l=1}^{n} e_{j l}(k) \tilde{x}_{l}(k)\left(\exp \left\{u_{l}(k)\right\}-1\right) \\
& -\sum_{l=1}^{m} p_{j l}(k) \tilde{y}_{l}(k)\left(\exp \left\{v_{l}(k)\right\}-1\right) .
\end{aligned}
$$

So,

$$
\begin{aligned}
\left|u_{i}(k+1)\right| \leq & \left|1-\left(c_{i}(k)+\frac{1}{a_{i}(k)}\right) r_{1 i}(k) \tilde{x}_{i}(k) \exp \left\{\theta_{i}(k) u_{i}(k)\right\}\right|\left|u_{i}(k)\right| \\
& +\frac{r_{1 i}(k) \tilde{x}_{i}(k)}{a_{i}^{2}(k)} \sum_{l=1, l \neq i}^{n} b_{i l}(k) \tilde{x}_{l}(k) \exp \left\{\theta_{l}(k) u_{l}(k)\right\}\left|u_{l}(k)\right| \\
& +\sum_{l=1}^{m} d_{i l}(k) \tilde{y}_{l}(k) \exp \left\{\xi_{l}(k) v_{l}(k)\right\}\left|v_{l}(k)\right| \\
\left|v_{i}(k+1)\right| \leq & \left|1-p_{j j}(k) \tilde{y}_{j}(k) \exp \left\{\xi_{j}(k) v_{j}(k)\right\}\right|\left|v_{j}(k)\right| \\
& +\sum_{l=1}^{n} e_{j l}(k) \tilde{x}_{l}(k) \exp \left\{\theta_{l}(k) u_{l}(k)\right\}\left|u_{l}(k)\right| \\
& +\sum_{l=1}^{m} p_{i l}(k) \tilde{y}_{l}(k) \exp \left\{\xi_{l}(k) v_{l}(k)\right\}\left|v_{l}(k)\right|,
\end{aligned}
$$

where $\theta_{l}(k), \xi_{l}(k) \in[0,1]$, to complete the proof, it suffices to show that

$$
\lim _{k \rightarrow+\infty} u_{i}(k)=0, \quad \lim _{k \rightarrow+\infty} v_{j}(k)=0 .
$$


In view of (3.1), we can choose $\varepsilon>0$ small enough such that

$$
\begin{aligned}
\lambda_{i}^{\varepsilon}= & \max \left\{\left|1-\left(c_{i}^{u}+\frac{1}{a_{i}^{l}}\right) r_{1 i}^{u}\left(M_{i}+\varepsilon\right)\right|,\left|1-\left(c_{i}^{l}+\frac{1}{a_{i}^{u}}\right) r_{1 i}^{l}\left(m_{i}-\varepsilon\right)\right|\right\} \\
& +\frac{r_{1 i}^{u}\left(M_{i}+\varepsilon\right)}{\left(a_{i}^{l}\right)^{2}} \sum_{l=1, l \neq i}^{n} b_{i l}^{u}\left(M_{l}+\varepsilon\right)+\sum_{l=1}^{m} d_{i l}^{u}\left(Q_{l}+\varepsilon\right)<1, \\
\delta_{j}^{\varepsilon}= & \max \left\{\left|1-p_{j j}^{u}\left(Q_{j}+\varepsilon\right)\right|,\left|1-p_{j j}^{l}\left(q_{j}-\varepsilon\right)\right|\right\}+\sum_{l=1}^{n} e_{j l}^{u}\left(M_{l}+\varepsilon\right) \\
& +\sum_{l=1}^{n} p_{j l}^{u}\left(Q_{l}+\varepsilon\right)<1 .
\end{aligned}
$$

For the above $\varepsilon>0$, according to Theorem 2.4 in Section 2, there exists a $k^{*} \in N$ such that

$$
\begin{array}{ll}
m_{i}-\varepsilon \leq x_{i}(k), & \tilde{x}_{i}(k) \leq M_{i}+\varepsilon, \\
q_{j}-\varepsilon \leq y_{j}(k), & \tilde{y}_{j}(k) \leq Q_{j}+\varepsilon,
\end{array}
$$

for all $k \geq k^{*}$.

Noticing that $\theta_{l}(k), \xi_{l}(k) \in[0,1]$ implies that $\tilde{x}_{l}(k) \exp \left\{\theta_{l}(k) u_{l}(k)\right\}$ lies between $\tilde{x}_{l}(k)$ and $x_{l}(k), \tilde{y}_{l}(k) \exp \left\{\xi_{l}(k) v_{l}(k)\right\}$ lies between $\tilde{y}_{l}(k)$ and $y_{l}(k)$. From (3.5), we get

$$
\begin{aligned}
\left|u_{i}(k+1)\right| \leq & \max \left\{\left|1-\left(c_{i}^{u}+\frac{1}{a_{i}^{l}}\right) r_{1 i}^{u}\left(M_{i}+\varepsilon\right)\right|,\left|1-\left(c_{i}^{l}+\frac{1}{a_{i}^{u}}\right) r_{1 i}^{l}\left(m_{i}-\varepsilon\right)\right|\right\}\left|u_{i}(k)\right| \\
& +\frac{r_{1 i}^{u}\left(M_{i}+\varepsilon\right)}{\left(a_{i}^{l}\right)^{2}} \sum_{l=1, l \neq i}^{n} b_{i l}^{u}\left(M_{l}+\varepsilon\right)\left|u_{l}(k)\right|+\sum_{l=1}^{m} d_{i l}^{u}\left(Q_{l}+\varepsilon\right)\left|v_{l}(k)\right|, \\
\left|v_{j}(k+1)\right| \leq & \max \left\{\left|1-p_{j j}^{u}\left(Q_{j}+\varepsilon\right)\right|,\left|1-p_{j j}^{l}\left(q_{j}-\varepsilon\right)\right|\right\}\left|v_{j}(k)\right| \\
& +\sum_{l=1}^{n} e_{j l}^{u}\left(M_{l}+\varepsilon\right)\left|u_{l}(k)\right|+\sum_{l=1}^{n} p_{j l}^{u}\left(Q_{l}+\varepsilon\right)\left|v_{l}(k)\right| .
\end{aligned}
$$

Let $\gamma=\max \left\{\lambda_{i}^{\varepsilon}, \delta_{j}^{\varepsilon}\right\}$, then $\gamma<1$. In view of (3.9), for $k \geq k^{*}$, we get

$$
\max \left\{\left|u_{i}(k+1)\right|,\left|v_{j}(k+1)\right|\right\} \leq \gamma \max \left\{\left|u_{i}(k)\right|,\left|v_{j}(k)\right|\right\} .
$$

This implies

$$
\max \left\{\left|u_{i}(k)\right|,\left|v_{j}(k)\right|\right\} \leq \gamma^{k-k^{*}} \max \left\{\left|u_{i}\left(k^{*}\right)\right|,\left|v_{j}\left(k^{*}\right)\right|\right\} .
$$


From (3.11), we have

$$
\lim _{k \rightarrow+\infty} u_{i}(k)=0, \quad \lim _{k \rightarrow+\infty} v_{j}(k)=0 .
$$

This ends the proof of Theorem 3.1.

\section{Existence and Stability of Periodic Solution}

In this section, we further assume that the coefficients of the system (1.1) satisfies (4.1).

There exists a positive integer $\omega$ such that for $k \in N$,

$$
\begin{array}{lc}
0<r_{1 i}(k+\omega)=r_{1 i}(k), & 0<b_{i l}(k+\omega)=b_{1 i}(k), \\
0<a_{i}(k+\omega)=a_{i}(k), & 0<c_{i}(k+\omega)=c_{i}(k), \\
0<r_{2 j}(k+\omega)=r_{2 j}(k), & 0<d_{i l}(k+\omega)=d_{i l}(k) \\
0<e_{j l}(k+\omega)=e_{j l}(k), & 0<p_{j l}(k+\omega)=p_{j l}(k) .
\end{array}
$$

Our first result concerned with the existence of a positive periodic solution of system

Theorem 4.1. Assume that (2.5) and (2.14) hold, then system (1.1) admits at least one positive w-periodic solution which ones denotes by $\left(\tilde{x}_{1}(k), \ldots, \tilde{x}_{n}(k), \tilde{y}_{1}(k), \ldots, \tilde{y}_{m}(k)\right)$.

Proof. As noted at the end of Section 2,

$$
D^{n+m}:=\left[m_{1}, M_{1}\right] \times \cdots\left[m_{n}, M_{n}\right] \times\left[q_{1}, Q_{1}\right] \times \cdots\left[q_{m}, Q_{m}\right]
$$

is an invariant set of system (1.1). Thus, we can define a mapping $F$ on $D^{n+m}$ by

$$
F\left(x_{1}(0), \ldots, x_{n}(0), y_{1}(0), \ldots, y_{m}(0)\right)=\left(x_{1}(\omega), \ldots, x_{n}(\omega), y_{1}(\omega), \ldots, y_{m}(\omega)\right)
$$

for $\left(x_{1}(0), \ldots, x_{n}(0), y_{1}(0), \ldots, y_{m}(0)\right) \in D^{n+m}$. Obviously, $F$ depends continuously on $\left(x_{1}(0), \ldots, x_{n}(0), y_{1}(0), \ldots, y_{m}(0)\right)$. Thus, $F$ is continuous and maps the compact set $D^{n+m}$ into itself. Therefore, $F$ has a fixed point. It is easy to see that the solution $\left(\tilde{x}_{1}(k), \ldots, \tilde{x}_{n}(k), \tilde{y}_{1}(k), \ldots, \tilde{y}_{m}(k)\right)$ passing through this fixed point is an $\omega$-periodic solution of the system (1.1). This completes the proof of Theorem 4.1.

Theorem 4.2. Assume that (2.5), (2.14), and (3.1) hold, then system (1.1) has a global stable positive w-periodic solution.

Proof. Under the assumption of Theorem 4.2, it follows from Theorem 4.1 that system (1.1) admits at least one positive $\omega$-periodic solution. Also, Theorem 3.1 ensures the positive solution to be globally stable. This ends the proof of Theorem 4.2. 


\section{References}

[1] P. Cull, "Global stability of population models," Bulletin of Mathematical Biology, vol. 43, no. 1, pp. 47-58, 1981.

[2] J. E. Franke and A.-A. Yakubu, "Geometry of exclusion principles in discrete systems," Journal of Mathematical Analysis and Applications, vol. 168, no. 2, pp. 385-400, 1992.

[3] Y. Huang, "A note on global stability for discrete one-dimensional population models," Mathematical Biosciences, vol. 102, no. 1, pp. 121-124, 1990.

[4] W. D. Wang and Z. Y. Lu, "Global stability of discrete models of Lotka-Volterra type," Nonlinear Analysis: Theory, Methods E Applications, vol. 35, no. 8, pp. 1019-1030, 1999.

[5] Y. Saito, W. Ma, and T. Hara, "A necessary and sufficient condition for permanence of a Lotka-Volterra discrete system with delays," Journal of Mathematical Analysis and Applications, vol. 256, no. 1, pp. 162 $174,2001$.

[6] M. P. Hassell and H. N. Comins, "Discrete time models for two-species competition," Theoretical Population Biology, vol. 9, no. 2, pp. 202-221, 1976.

[7] R. M. May, Theoretical Ecology, Principles and Applications, Sounders, Philadelphia, Pa, USA, 1976.

[8] L. Bai, M. Fan, and K. Wang, "Existence of positive periodic solution for difference equations of a cooperative system," Journal of Biomathematics, vol. 19, no. 3, pp. 271-279, 2004 (Chinese).

[9] F. Chen, "Permanence and global attractivity of a discrete multispecies Lotka-Volterra competition predator-prey systems," Applied Mathematics and Computation, vol. 182, no. 1, pp. 3-12, 2006.

[10] H.-F. Huo and W.-T. Li, "Permanence and global stability for nonautonomous discrete model of plankton allelopathy," Applied Mathematics Letters, vol. 17, no. 9, pp. 1007-1013, 2004.

[11] L. Wang and M. Q. Wang, Ordinary Difference Equation, Xin-jiang University Press, Xin-jiang, China, 1991.

[12] F. Chen, "Permanence of a discrete $n$-species food-chain system with time delays," Applied Mathematics and Computation, vol. 185, no. 1, pp. 719-726, 2007. 


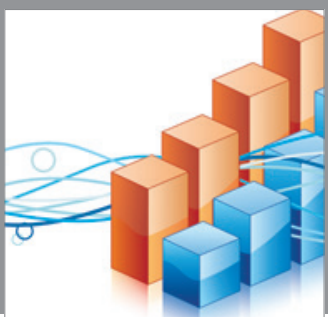

Advances in

Operations Research

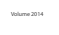

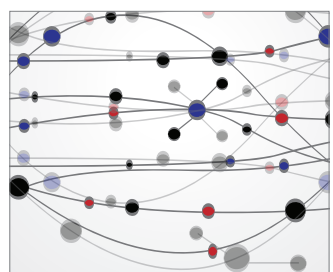

\section{The Scientific} World Journal
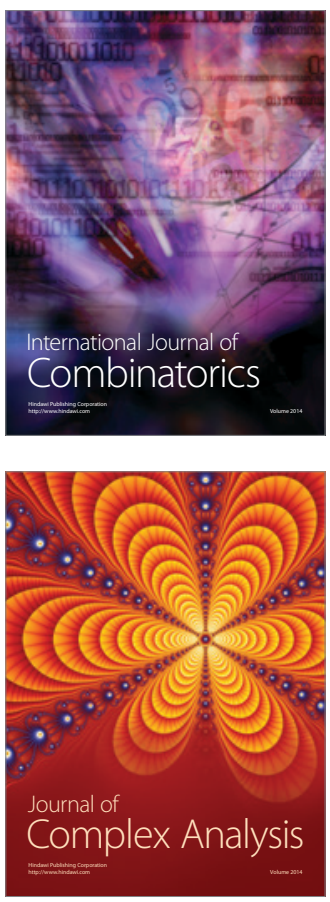

International Journal of

Mathematics and

Mathematical

Sciences
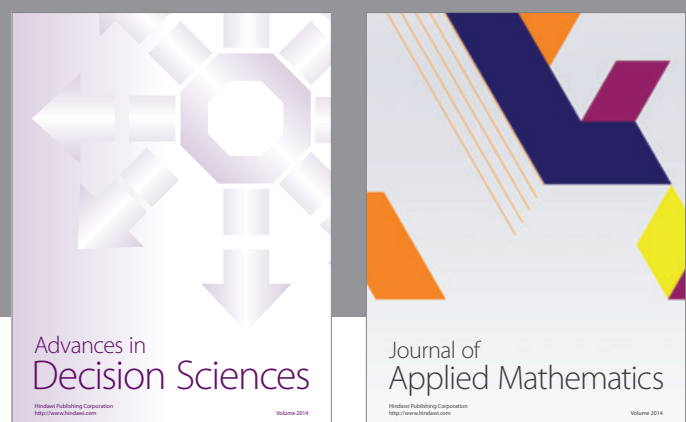

Journal of

Applied Mathematics
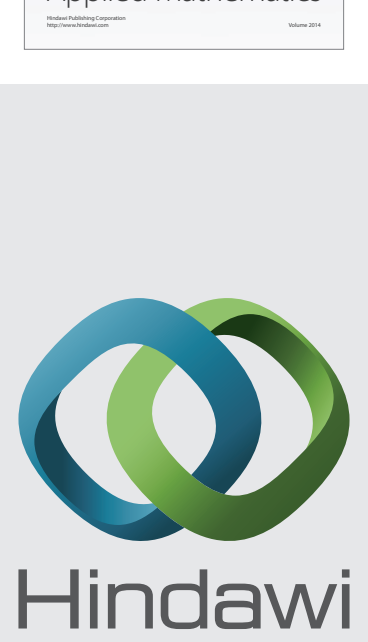

Submit your manuscripts at http://www.hindawi.com
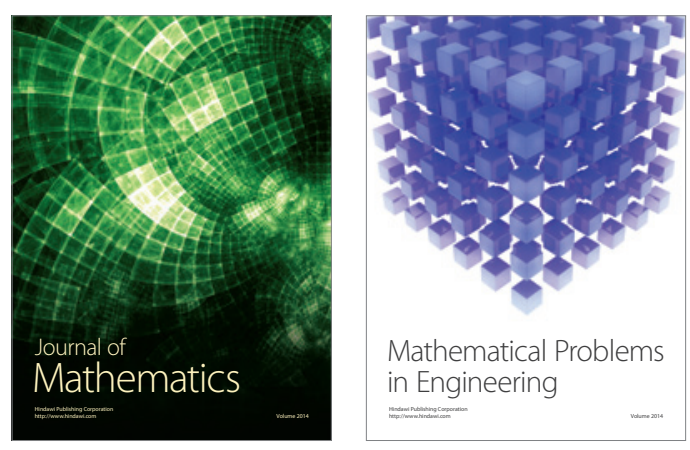

Mathematical Problems in Engineering
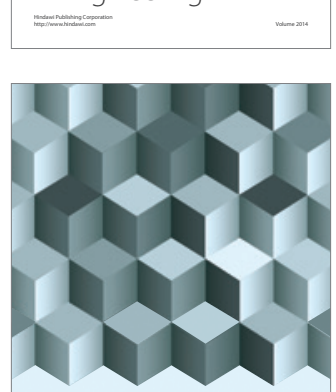

Journal of

Function Spaces
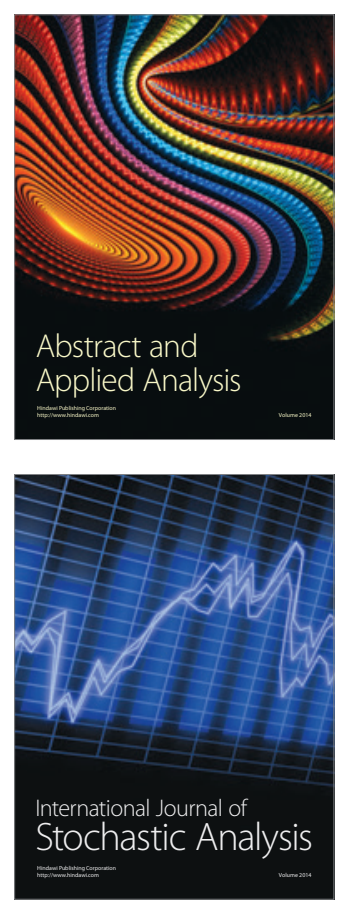

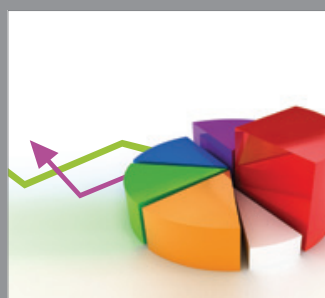

ournal of

Probability and Statistics

Promensencen
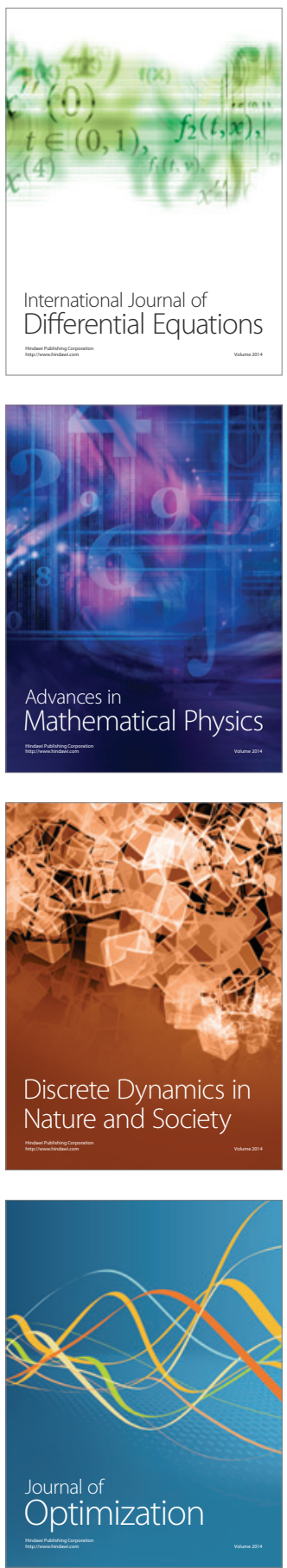